\title{
The Mitochondrial DNA of Xenoturbella bocki: Genomic Architecture and Phylogenetic Analysis
}

\author{
Marleen Perseke - Thomas Hankeln - Bettina Weich · Guido Fritzsch · Peter F. \\ Stadler · Olle Israelsson · Detlef Bernhard · Martin Schlegel
}

Received: ** April 2007 / Accepted: **********

\begin{abstract}
The phylogenetic position of Xenoturbella bocki has been a matter of controversy since its description in 1949. We sequenced a second complete mitochondrial genome of this species and performed phylogenetic analyses based on the amino-acid sequences of all 13 mitochondrial proteincoding genes and on its gene order. Our results confirm the deuterostome relationship of Xenoturbella. However, in contrast to a recently published study [Bourlat et al., Nature 444: 85-88 (2006)], our data analysis suggests a more basal branching of Xenoturbella within the deuterostomes, rather than a sister-group relationship to the Ambulacraria (Hemichordata and Echinodermata).
\end{abstract}

Keywords Xenoturbella, phylogeny, mitochondrial genome, basal deuterostomes

The project was funded by the Deutsche Forschungsgemeinschaft under the auspices of SPP-1174 "Deep Metazoan Phylogeny", Projects HA 2103/4-1, SCHL 229/14-1, STA 850/2-1, and STA 850/3-1.

Detlef Bernhard, Marleen Perseke, Martin Schlegel

Institut für Zoologie, Molekulare Evolution und Systematik der Tiere Universität Leipzig, Talstr. 33, 04103 Leipzig, Germany

Tel: ++49 3419736732 , Fax: ++493419736789

E-mail: $\{$ perseke,bernhard,schlegel\}@ @r.uni-leipzig.de

Thomas Hankeln, Bettina Weich

Institut für Molekulargenetik, Johannes Gutenberg Universität Mainz, J. J. Becherweg 32, D-55099 Mainz, Germany

E-mail: \{hankeln,weich\}@uni-mainz.de

Guido Fritzsch, Peter F. Stadler

Interdisziplinäres Zentrum für Bioinformatik, Universität Leipzig, Härtelstr. 16-18, 04107 Leipzig, Germany

E-mail: fritzsch@izbi.uni-leipzig.de

Peter F. Stadler Lehrstuhl für Bioinformatik, Institut für Informatik, Universität Leipzig, Härtelstr. 16-18, 04107 Leipzig, Germany

E-mail: studla@bioinf.uni-leipzig.de·Olle Israelsson

Museum of Evolution, Uppsala University, Norbyvagen 16, S-75236

Uppsala, Sweden

E-mail: olle.israelsson@bredband.net

\section{Introduction}

Xenoturbella bocki is a small, up to $4 \mathrm{~cm}$ long, free-living marine animal with an irregular and flattened shape (Israelsson, 1999). Its unusual morphology with an epithelial epidermis and gastrodermis, but without anus and only a "statocyst" as distinct organ (Ehlers, 1991), led to highly divergent phylogenetic interpretations. Indeed, the phylogenetic position of Xenoturbella bocki has been a matter of controversy since its description by Westblad (1949). Initially, most authors grouped Xenoturbella within the Protostomia. Histological data (Westblad, 1949) suggested Xenoturbella to be a new taxon within the Acoelomorpha, a hypothesis that was later supported by ultrastructural features of the epidermal cilia (Franzén and Afzelius, 1987; Rohde et al., 1988; Rieger et al., 1991; Lundin, 1998). Other morphological (Ehlers, 1991), ontogenetic (Israelsson, 1997, 1999; Israelsson and Budd, 2005), and ultrastructural features (Raikova et al., 2000), however, casted serious doubt on the acoelomorpha hypothesis. Instead, Xenoturbella was variably grouped to enteropneusts or holothurians (Reisinger, 1960), to bryozoans (Zrzavy et al., 1998), and to molluscs (Israelsson, 1997, 1999). The immunoreactivity of $X$. bocki nerve cells to antibodies raised against echinoderm neuropeptides suggested a close phylogenetic relationship to Ambulacraria (Stach et al., 2005). Other authors advocated a basal position within the metazoa (Jägersten, 1959; Ehlers and Sopott-Ehlers, 1997a; Raikova et al., 2000). With the realization that Acoela (and Nemertodermatida) are most likely basal bilaterians rather than protostomes (Ruiz-Trillo et al., 2002; Telford et al., 2003; RuizTrillo et al., 2004), the basal metazoa hypothesis may, however, not be as much at odds with the acoelomorpha hypothesis as previously thought.

First molecular analyses of gene sequences placed Xenoturbella within bivalve molluscs (Noren and Jondelius, 1997), an analysis that was later recognized as flawed due to con- 


UAS I UAS II
( $336 \mathrm{bp})$
$(40 \mathrm{bp})$

Fig. 1 Linear mitochondrial genome map for Xenoturbella bocki. The gene order is arranged 5' -3'. Genes transcribed on the opposite strand are indicated by dark underlines. Numbers at gene boundaries indicate non-coding nucleotides between genes as inferred from sequence comparison. Negative numbers indicate gene overlaps of the corresponding size. Unassigned sequences (UAS) larger than 30 bp are shown by shaded areas. Gene name abbreviations: CxI/CxII/CxIII, cytochrome c oxidase subunit I/II/III; A8/A6, ATPase subunit 8/6; N1/N2/N3/N4/N4L/N5/N6, NADH dehydrogenase subunit 1/2/3/4/4L/5/6; CyB, cytochrome b; ssu, small subunit (12S) ribosomal RNA; and lsu, large subunit (16S) ribosomal RNA; tRNA genes are represented by their single-letter codes.

taminations of ingested prey (Bourlat et al., 2003). Authentic nuclear SSU rDNA sequences, mitochondrial proteincoding gene sequences and expressed sequencing tags (ESTs) supported a close relationship to Deuterostomia and placed Xenoturbella as the sister group of Ambulacraria (Hemichordata and Echinodermata) (Bourlat et al., 2003, 2006).

In this contribution we report on the sequencing of a second mitochondrial genome of Xenoturbella bocki, a detailed analysis of its gene order, and a re-evalution of the phylogenetic position of Xenoturbella based on mitochondrial protein sequences.

\section{Materials and Methods}

\subsection{Specimens, Sequencing, and Gene Identification}

Large specimens of Xenoturbella bocki were collected in Gullmarsfjorden, Bohuslän, Sweden in 2003. Total DNA was prepared from tissue of RNAlater-preserved specimen by standard phenol-chloroform extraction following proteinase $\mathrm{K}$ digestion. Long PCR primers were designed using known sequences of the mt genes CoxI and CoxII (Bourlat et al., 2003). Long PCR Enzyme Mix Kit (Fermentas) was used under recommended conditions to amplify the whole mitogenome. The amplification products were sequenced directly by primer walking using ABI Prism 3100 (Leipzig) and 3730 (GENterprise, Mainz) automated sequencers and BigDye Termination v3.1 Cycle Sequencing Kit (Applied Biosystems) in both directions. Additional starting points for faster primer walking were obtained by preparing a "mini" DNA library using four-cutter restriction enzymes and pUC1 8 plasmid vector. A complete list of primer sequences can be found in the Electronic Supplement. ${ }^{1}$

Protein and rRNA genes were identified by blastn (Altschul et al., 1997) database searches at NCBI. 19 of 22 tRNA genes were identified by the tRNAscan-SE program (Lowe and Eddy, 1997). The tRNA genes for Leucine (UUR), Serine (AGY), and Alanine were identified from se-

\footnotetext{
1 http://www.bioinf.uni-leipzig.de/Publications/ SUPPLEMENTS/07-009/
}

quence alignments and RNA secondary prediction using the Vienna RNApackage, version 1.6.1 (Hofacker et al., 1994).

The complete annotated sequence of the $X$. bocki mitogenome has been deposited in GenBank (AM296016).

\subsection{Phylogenetic Analysis}

We prepared three distinct data sets for phylogenetic analysis. In order to allow direct comparison and re-assessment of the results of Bourlat et al. (2006), the Xenoturbella bocki mtDNA sequence was aligned to an additional 31 complete mitochondrial genome sequences belonging to 19 deuterostomes, nine protostomes, and two cnidarian species, as well as the Xenoturbella sequence published by Bourlat et al. (2006). This taxon selection, referred to as "Set A", is essentially the same as the one studied by Bourlat et al. (2006).

We then expanded the data set to include additional basal metazoans, using poriferan sequences to root the tree. This "set B" also includes Trichoplax adhaerens. We tried to expand the representation of Protostomia to include a nematode. Even Trichinella spiralis (Lavrov and Brown, 2001), a relatively basal nematode with normally structured tRNAs, however, exhibited an extremely long branch, suggesting its inclusion would cause long branch attraction artefacts rather than improving the phylogenetic information.

A third set $\mathbf{C}$ was compiled comprising only of nine of the thirteen protein coding mitochondrial genes to allow the inclusion of the partial mitochondrial genome of Paratomella rubra (Ruiz-Trillo et al., 2004). This allowed us to test the deuterostome affinities of Xenoturbella in relation to a possible position as a basal bilaterian. All accession numbers of sequences used in this work are compiled in the electronic supplement.

All sequences of the protein coding mitochondrial genes were aligned separately using T-Coffee with default parameters (Notredame et al., 2000). We chose T-Coffee, which generates multiple alignments based on a library produced by using a mixture of local and global pair-wise alignment, because this program has been demonstrated to yield more accurate alignments in particular in difficult case with large divergences times of the taxa (Notredame et al., 2000). 
The color scheme and the implemented CORE index of $\mathrm{T}$-Coffee were used as an indicator of the reliability of the alignment. Multiple alignments were then trimmed on the first and the last constant site. The concatenated matrix included the trimmed alignments of the protein coding genes with an alignment quality index over 40 , a threshold that was reached by all thirteen aligned proteins.

Sequence evolution models and parameters were tested separately for each protein sequence separately and for the concatenated data set using ProtTest version 1.3 (Abascal et al., 2005). The selected model was the most general, reversible Markov model of mitochondrial amino acids: MtRev model (Adachi and Hasegawa, 1996) with a gamma shape (4 rate categories) parameter of $\gamma=0.711$ and a proportion of invariable sites of 0.115 . These parameters were then used for phylogenetic reconstructions. The analyses were performed with the program PAUP* (Swofford, 2002) for neighbor joining and maximum parsimony, with TREE-PUZZLE, version 5.2, (Schmidt et al., 2002) for maximum likelihood and with MrBayes, version 3.1.2 (Ronquist and Huelsenbeck, 2003), and PhyloBayes, version 2.1 (Lartillot and Philippe, 2004, 2006) for bayesian analysis.

To test the robustness of bifurcations, bootstrap analysis (10000 replicates for NJ, 2000 replicates for MP, and 1000 puzzling steps for ML) was calculated. Bayesian analysis was run with the best-fit model as inferred by ProtTest for 1000000 generations, with a sampling frequency of 10 generations. From the 100000 trees found, we determined a subset of trees for building the consensus tree by inspecting the likelihood values of trees saved by MrBayes. The burnin was set to 25000 trees to ensure that stable likelihood values were achieved.

For the data sets $\mathbf{B}$ and $\mathbf{C}$ we calculated the bayesian analysis with mixture configuration of the substitution model CAT (Lartillot and Philippe, 2004) with a free number of categories and continuous gamma distribution. Four chains with 1000 sample trees (sample frequency 1) were run. The default cutoff of 0.05 was used to include only partitions with maximum probability.

\subsection{Analysis of Gene Order}

Mitochondrial gene orders (including all mRNA, rRNA and tRNA genes) were compared using circal (Fritzsch et al., 2006), which calculates distances based on a circular list alignment of the mitochondrial genome order in spite of differences and gene content. Different weights are used by this program for rearranged blocks depending on whether they contain proteins, only rRNAs, or only tRNAs. In addition, we manually compared the gene order of Xenoturbella with representative basal deuterostomes.

\section{Results}

\subsection{Characteristics of the Xenoturbella Mitogenome}

The $X$. bocki mt genome sequenced in our analyses (GenBank $\boldsymbol{A M 2 9 6 0 1 6}$ ) spaned 15,232 bp and contained all typical metazoan mt genes: 13 proteins, 2 rRNA, and 22 tRNA genes. Most genes were encoded on the same strand, except for the ND6 gene and seven tRNA genes (Fig. 1).

Only few differences were found when comparing our data to the mtDNA sequence from Xenoturbella bocki $(15,234$ bp) sequenced by Bourlat et al. (2006), Genbank accession NC_008556. Both nucleotide sequences revealed a compact organization of Xenoturbella mtDNA with only $2.4 \%$ of noncoding sequence. Most of the non-coding nucleotides resided in a single large "unassigned sequence" (UAS I, 336 bp in our sequence and 2nt shorter in the genome sequences by Bourlat et al. (2006)) flanked by tRNALeu (CUN) and ND6, and an additional smaller UAS region (UAS II, 40 bp) flanked by the tRNAGlu and ND1 genes. A total of 73 nucleotide differences were observed between the two available sequences. The putative control region UAS I was found to be slightly enriched for differences between both mtDNA versions $(3.3 \%$, including two additional nucleotides in our sequence) compared to the whole sequence $(0.5 \%)$. Within the protein-coding genes, we observed 39 differences (excluding sequencing ambiguities, i.e., characters such as $\mathrm{N}$ or $\mathrm{Y}$ ); 15 of these substitutions were non-synonymous causing amino acid replacements.

The reading frames of all protein coding-genes were delimited by the start codons ATG or GTG and complete termination codons. However, two possible start codons were found for ND4L, CoxII and ND5.

Due to the uncertain phylogenetic position of Xenoturbella, different translation tables were tested to translate the gene sequences into amino acid sequences. All mitochondrial translation tables of deuterostomes (invertebrate, echinoderm or vertebrate code) are possible, in the sense that none of them implied a premature termination within the coding regions. Analyses using the gendecoder server (Abascal et al., 2006) predicted the generic invertebrate mitochondrial code, which then was used throughout the subsequent analysis. In particular, there was no evidence that Xenoturbella shares any one of the codon reassignments characteristic for Hemichordata (Castresana et al., 1998a) and/or Echinodermata (Himeno et al., 1987). All 15 differences between the amino-acid sequences reported by Bourlat et al. (2006) and this study, resp., were based on nucleotide differences (see above) and did not depend on the choice of translation table.

The mitochondrial genome map of Xenoturbella bocki, (Fig. 1) was found the be largely in accordance with the gene orders of other representatives of Deuterostomia. In particular, we compared the gene order of X. bocki with the hemi- 

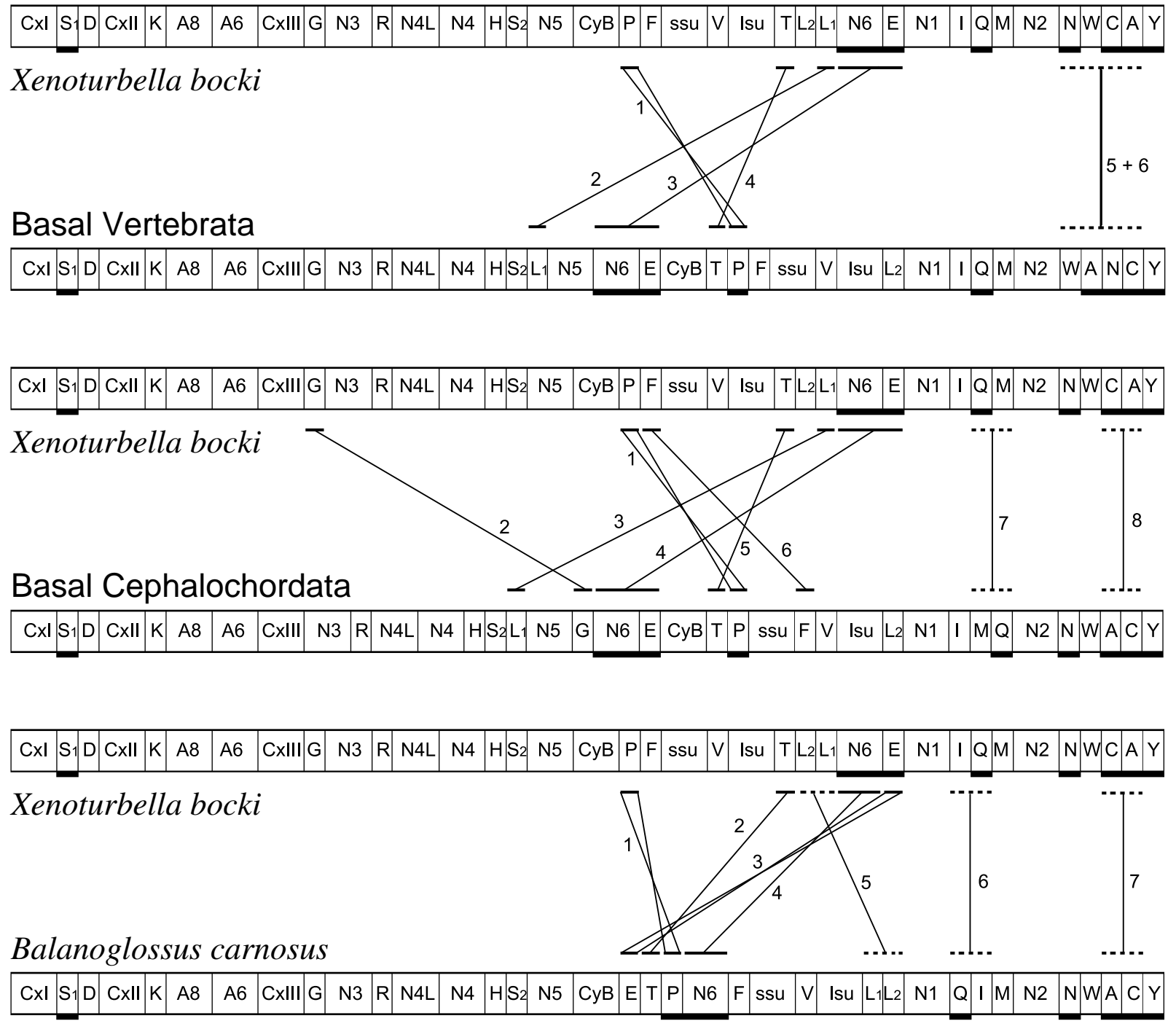

Fig. 2 The mitochondrial gene order of $X$. bocki compared to mitochondrial gene orders of other basal deuterostomes: consensus gene order of vertebrates (Boore, 1999), hemichordate gene order represented by Balanoglossus carnosus (Castresana et al., 1998b), inferred ancestral gene order of cephalochordates (Nohara et al., 2005). As in Fig. 1, gene order is 5'-3'. Genes transcribed from from the opposite strand are underlined. For gene name abbreviations see caption of Fig. 1. Translocations are marked by straight lines, and inversions are marked by cross lines. Changes of the position between joining tRNAs are shown by interrupted lines. See text for detailed discussion.

chordate Balanoglossus carnosus (Castresana et al., 1998b), the reconstructed basal gene order of cephalochordates derived by Nohara et al. (2005), and to the consensus gene order of vertebrates (Boore, 1999) (Fig. 2). In contrast, protostome gene arrangements (Boore, 1999) consistently exhibited much larger differences (data not shown).

The transformation of the gene order of $X$. bocki to the closest representative of the different deuterostome groups required the inversion of tRNAPro (Fig. 2, cross line 1) and nearly the same number of additional steps: five relocations to the consensus gene order of vertebrates (tRNALeu(CUN), ND6-tRNAGlu, tRNAThr, tRNACys and tRNAAsn or tRNAAla and tRNATrp), six relocations to the gene order of
Balanoglossus carnosus (tRNAThr, ND6, tRNAGlu, tRNACys or tRNAAla, tRNALeu(CUN) or tRNALeu(UUR), tRNAIle or tRNAGln) and seven relocations to the gene order of basal cephalochordates (tRNAGly, ND6-tRNAGlu, tRNALeu(CUN), tRNAPhe, tRNAThr, tRNACys or tRNAAla, tRNAMet or tRNAAsn).

Hence, the gene arrangement of $X$. bocki shared features with all three groups, e.g., the fragment ND6-tRNAGlu was also present in vertebrates and cephalochordates but was lacking in hemichordates. In contrast, the location of the tRNAGly in Xenoturbella was identical to Hemichordata and Vertebrata while the order within the fragment tRNAAsn- 


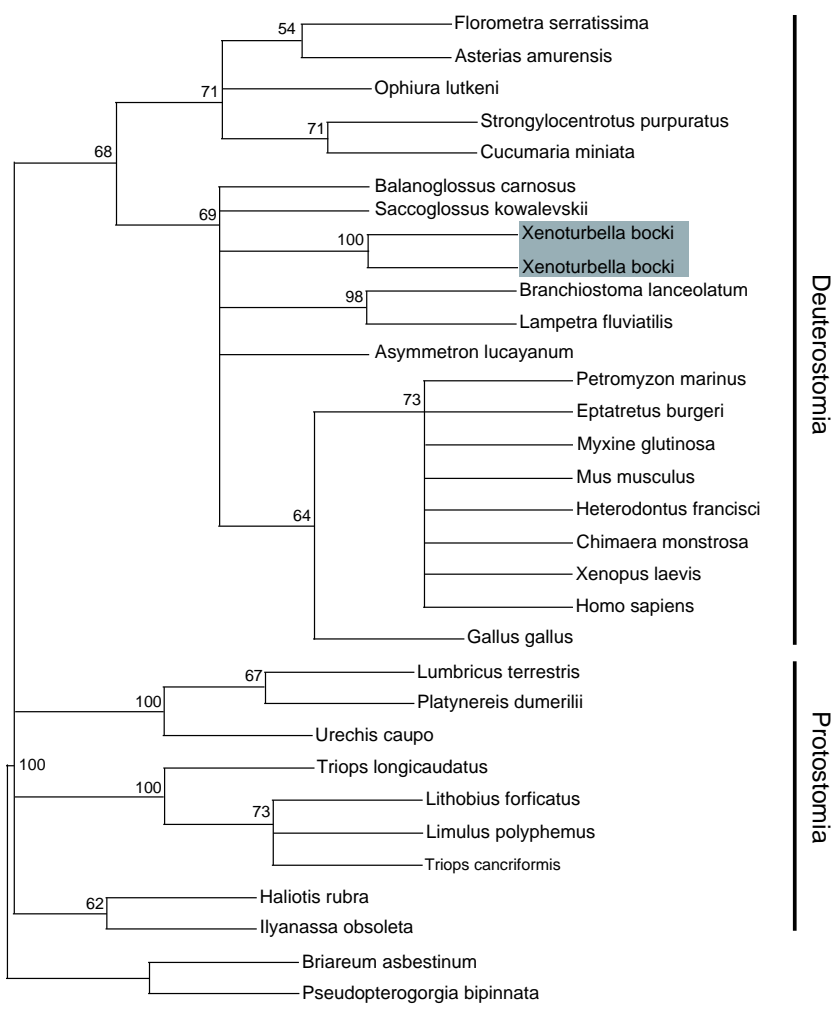

Fig. 3 Maximum parsimony analysis of the mitochondrial gene order using the program circal (Fritzsch et al., 2006). Numbers at the nodes represent bootstrap values of MP analysis with heuristic search (2000 replicates).

tRNATrp-tRNACys-tRNAAla-tRNATyr is more similar to Hemichordata and Cephalochordata than to Vertebrata.

Not surprisingly, therefore, the analysis of the mitochondrial gene orders using circal (Fritzsch et al., 2006) resulted in a tree topology in which all deuterostome taxa form a common branch (Fig. 3) while the protostomes branch off in a polytomy. The first branch within the deuterostomes is formed by the echinoderms followed by an unresolved polytomy consisting of X. bocki, Hemichordata, Cephalochordata, and Vertebrata (Fig. 3).

A comparison of the gene order of $X$. bocki with that of the partial mitogenome of the acoel Paratomella did not reveal any conspicuous similarities that might place those species into closer proximity.

Lavrov and Lang (2005) reconstructed a hypothetical ancestral mitochondrial gene order of Deuterostomia and demonstrated that this arrangment was most likely very similar to that of ancestral bilaterian. If correct, their reconstruction implies that gene order rules out any affinities of Xenoturbella to protostomes, but at the same time makes it unlikely that gene order alone can be used to distinguish between a basal position within the deuterostomes and a basal bilaterian position.

\subsection{Phylogenetic Analysis of Protein Coding Genes}

We used the amino acid sequences of all 13 protein-coding genes to examine the phylogenetic relationship of Xenoturbella, including both our own mitogenome sequence and the sequence reported by Bourlat et al. (2006).

We analyse here three datasets. Set A was most similar to the one analyzed by Bourlat et al. (2006). Deviating from their data set, we included two representatives of diploblast metazoans as outgroups, but excluded the Urochordata (Tunicata) because our initial analyses (data not shown) always showed them basal to all other bilaterians. This exceptional result was observed repeatedly in previous studies (Yokobori et al., 2003, 2005; Bourlat et al., 2006) and can be explained by particularly rapid sequence evolution of urochordate $\mathrm{mt}$ genes leading to long-branch attraction.

All phylogenetic methods yielded the same tree topology regarding the main taxa and clearly recovered Protostomia, Deuterostomia, Ambulacraria (Echinodermata, Hemichordata) and Chordata (Cephalochordata, Vertebrata) as monophyletic groups. However, we also noted that within vertebrates and echinodermates some unstable or unusual branchings occur. Our analyses always resulted in a conjoint placement of both Xenoturbella isolates at the base of the deuterostomes followed by a split into the Ambulacraria on the one hand, and the Cephalochordata and Vertebrata on the other hand. This basal branching of Xenoturbella was supported by a bayesian posterior probability of 1.0, a quartet puzzling support value of $73 \%$ (ML), and bootstrap values of $68 \%$ (MP) and $84 \%$ (NJ). The full trees as well as support values for all nodes are compiled in the electronic supplement.

For a comparison to the analyses of Bourlat et al. (2006), we also calculated trees in which the four amino acids methionine, isoleucine, asparagine, and lysine ( $\mathrm{M}, \mathrm{I}, \mathrm{N}, \mathrm{K})$ were converted into "missing data" to avoid possible influences due to codon reassignments in the Ambulacrarian mitogenomes (Himeno et al., 1987; Castresana et al., 1998a). For details on the computational procedure we refer to the work by Bourlat et al. (2006). Bayesian, NJ, and MP analyses again resulted in the same basal placement of both Xenoturbella isolates, while ML analyses even showed Xenoturbella at the base of the Metazoa (data not shown). Compared to our initial analyses, which take all amino acids into account (Fig. 4), all support values with the exception of the ML analysis were reduced in the partially converted dataset: bayesian posterior probability $=0.89$, quartet puzzling support $=89 \%(\mathrm{ML})$, bootstrap support values $=61 \%(\mathrm{MP})$ and $50 \%(\mathrm{NJ})$.

Set $\mathbf{B}$ further expanded the taxon sampling to include further outgroup species (Trichoplax adhaerens and two poriferans). The results were very similar to that of set A: Fig. 4 shows the tree obtained using the PhyloBayes program. 


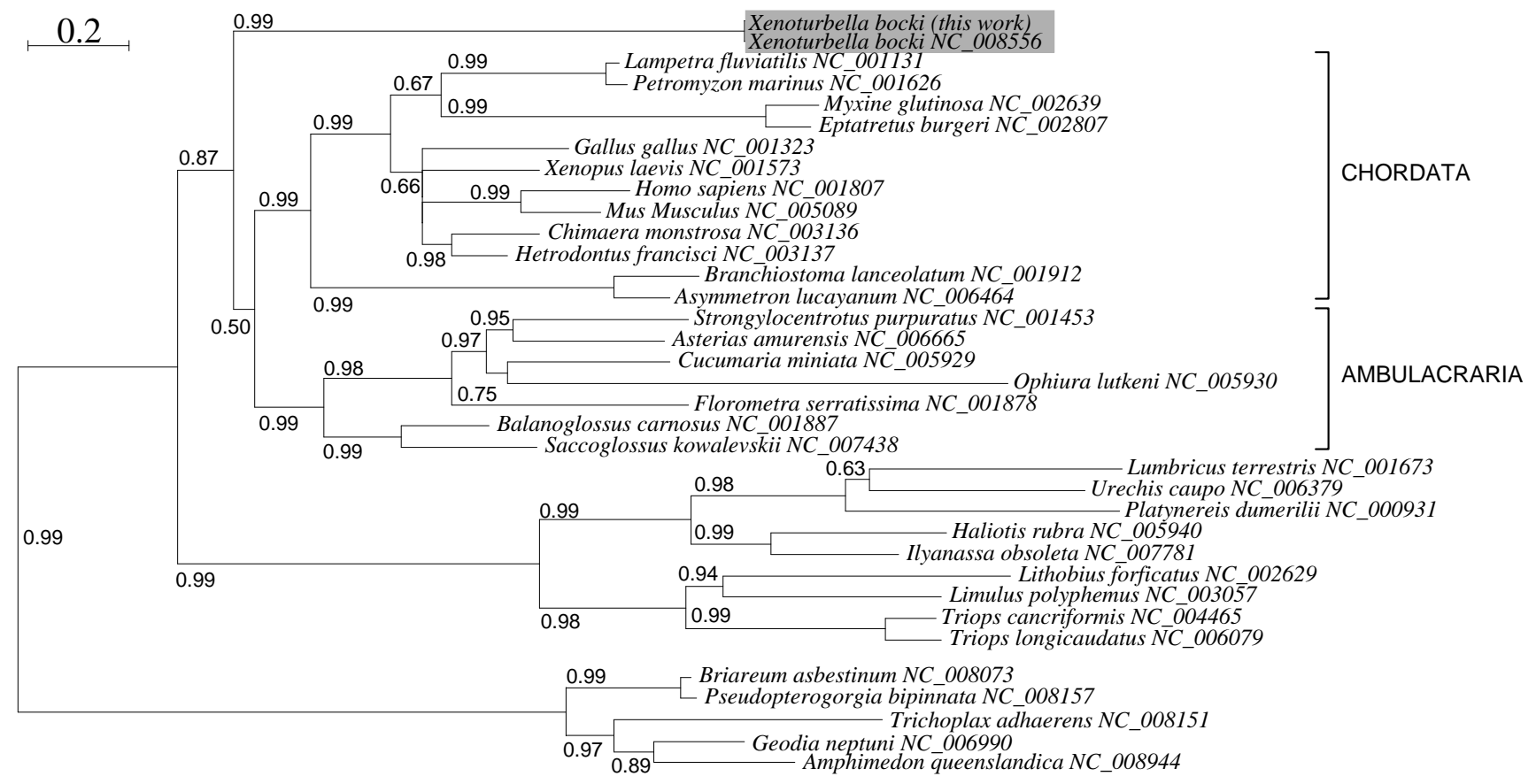

Fig. 4 Analysis of concatenated mitochondrial amino-acid using PhyloBayes and the CAT model with a free number of categories and continuous gamma distribution. The tree is rooted at the two poriferan sequences. Numbers at the nodes indicate Bayesian posterior probabilities; scale units are substitutions per site.

Set $\mathbf{C}$ was chosen to test possible affinities of Xenoturbella with basal bilaterians. Hence we included Paratomella rubra, the only acoelomorph for which at least a partial mitochondrial genome has been sequenced so far. In contrast to the two other data sets, set $\mathbf{C}$ favored a position of Xenoturbella bocki among basal bilaterians. However, the branch leading to Paratomella was unusally long (see electronic supplement for tree and support values), suggesting that the grouping of Xenoturbella with Paratomella could be a long branch attraction artifact. In constrast to the Bayesian analysis, maximum parsimony showed Xenoturbella again as a basal deuterostome. The results from the different data sets and methods are summarized in Fig. 5.

\section{Discussion}

The analysis of mitochondrial protein-coding genes clearly suggests that Xenoturbella belongs to the deuterostomes. This result is consistent with the analysis of the gene order by both visual analysis of break points and automated analysis using circal.

It is worth noting in this context that the mitochondrial data in work of Bourlat et al. (2006) place Xenoturbella as the sistergroup of Ambulacraria only when the "problematic" amino acids $\mathrm{M}, \mathrm{I}, \mathrm{N}, \mathrm{K}$, i.e., those that are conceivably affected by the differences in the mitochondrial genetic codes where treated as missing data, and when urochordates were included, which then assume the basal deuterostome
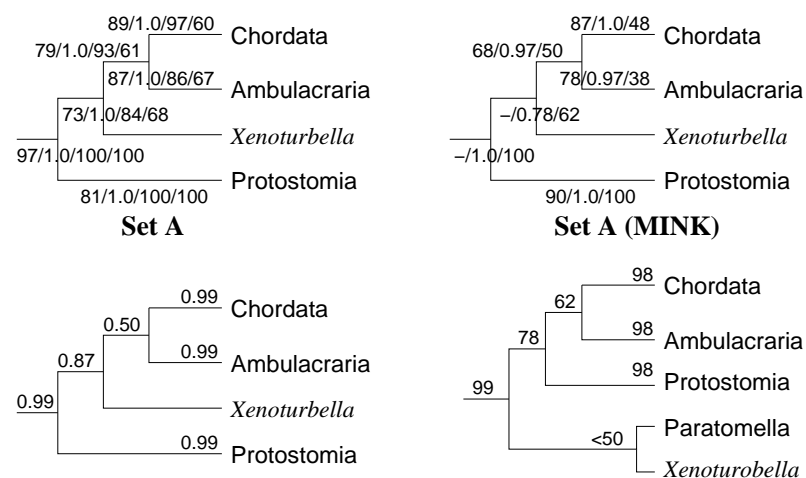

Set B

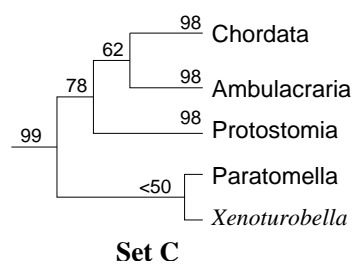

Fig. 5 Summary of phylogenetic analyses with different data sets and methods. Since the datasets are fairly similar, not all methods were applied to all data sets to save computer resources. Complete trees are provided in the electronic supplement. For set $\mathbf{A}$ support values are given in the format ML/bayesian/NJ/MP, with Bayesian posterior probabilities computed by MrBayes. Set A (MINK) refers to the variant of the analysis in which the amino acids $\mathrm{M}, \mathrm{I}, \mathrm{N}, \mathrm{K}$ are treated as missing data, showing ML/Bayesian/NJ support. ML results favor Xenoturbella in a basal bilaterian position. For sets $\mathbf{B}$ and $\mathbf{C}$ only Bayesian posterior probabilities were computed.

position. After exclusion of the urochordate sequences, which are likely to be affected by long branch effects do to their extremely high rates of evolution, the (Xenoturbella, Ambulacraria) affiliation cannot be reproduced in our hands even when M, I, N, K is treated as in Bourlat et al. (2006). 
While the position of Xenoturbella within deuterostomes cannot be resolved unambiguously, the available evidence, in particular the phylogenetic analysis of the mitochondrial protein sequences, points to a basal position within deuterostomes. While the hypothesis favoured by Bourlat et al. (2006), namely a position basal to the Ambulacraria (Echinodermata and Hemichordata), cannot be strictly ruled out based on our analysis, it is not supported by any positive evidence derivable from our data. On the contrary, a few of our analyses suggest, albeit with weak support, an even more basal position within the bilateria, possibly as the sistergroup the Eubilateria (Protostomia+Deuterostomia).

The result of our molecular study is in better agreement with most morphological studies, as it suggest a more basal position of Xenoturbella. Even though the morphology of Xenoturbella shares similarities to hemichordates, this evidence is far from unambiguous: Similarities in epidermis and sub-epidermal ECM (Reisinger, 1960) later have been interpreted as superficial (Pedersen and Pedersen, 1986, 1988). Similarities in myocytes and their junction are described as convergences and symplesiomorphy, respectively, by Ehlers and Sopott-Ehlers (1997b).

Eggs and embryos in Xenoturbella (Israelsson, 1997, 1999; Israelsson and Budd, 2005) posesses several protostome features, in particular a non-radial cleavage, the absence of cortical reaction during fertilization, and the ciliary ultrastructure. There are, on the other hand, possible similarities in constitution to echinoderm oocytes. Israelsson and Budd (2005) therefore suggest that Xenoturbella may have diverged from the other deuterostomes before the deuterostomes acquired their typical deuterostome features. The nervous system is also most compatible with a basal position, even if some similarities to deuterostome exist (Raikova et al., 2000). Therefore, the plesiomorphy of the morphological similarities between Xenoturbella and Ambulacraria cannot be ruled out.

The difficulties and contradicting evidence regarding the phylogenetic position of Xenoturbella is reminiscent of the ongoing discussion about the phylogenetic position of C. elegans, reviewed in the Wormbook (Fitch, 2005). There, the situation is far from resolved despite a finished genome and the availability of a much denser taxon sampling of Nematoda and their putative relatives compared to what we could hope for in the case of Xenoturbella. In addition to morphological information and the study of multi-gene sequence data - such as those from the mitogenome reported here and by Bourlat et al. (2006) and the EST-based analysis by Bourlat et al. (2006) - phylogenetic information can also be derived from complex molecular characters. The mitochondrial gene order is one such character (Sankoff et al., 1992). The hypothetical ancestral deterostome mitogenome gene order reconstructed by Lavrov and Lang (2005) is unfortunately very similar to that of the ancestral bilaterian. Xenoturbella does not share characteristic features with Pro- tostomia. A basal position within deuterostomes and a basal bilaterian position therefore cannot be distinguished on the basis of mitochondrial gene order. Furthermore, there is no significant part of the gene order shared between Xenoturbella and Ambulacraria that would place them together to the exclusion of Chordata.

\section{Supplemental Material}

Primer sequences, accession numbers of all mitogenomes used in this study, and trees reconstructed for sets $\mathbf{A}$ and $\mathbf{C}$ are provided at http://www.bioinf .uni-leipzig.de/ Publications/SUPPLEMENTS/07-009/.

\section{References}

Abascal F, Zardoya R, Posada D, 2005. ProtTest: Selection of best-fit models of protein evolution. Bioinformatics 21:2104-2105.

Abascal F, Zardoya R, Posada D, 2006. GenDecoder: genetic code prediction for metazoan mitochondria. Nucleic Acids Research 34:389-393.

Adachi J, Hasegawa M, 1996. Model of amino acid substitution in proteins encoded by mitochondrial DNA. J Mol Evol 42:459-468.

Altschul SF, Madden TL, Schaffer AA, Zhang J, Zhang Z, Miller W, Lipman DJ, 1997. Gapped BLAST and PSI-BLAST: a new generation of protein database search programs. Nucleic Acids Res 25:3389-3402.

Boore JL, 1999. Animal mitochondrial genomes. Nucleic Acids Res 27:1767-1780.

Bourlat SJ, Juliusdottir T, Lowe CJ, Freeman R, Aronowicz J, Kirschner M, Lander ES, Thorndyke M, Nakano H, Kohn AB, Heyland A, Moroz LL, Copley RR, Telford MJ, 2006. Deuterostome phylogeny reveals monophyletic chordates and the new phylum xenoturbellida. Nature 444:85-88.

Bourlat SJ, Nielsen C, Lockyer AE, Littlewood DT, Telford MJ, 2003. Xenoturbella is a deuterostome that eats molluscs. Nature 424:925-928.

Castresana J, Feldmaier-Fuchs G, Pääbo S, 1998a. Codon reassignment and amino acid composition in hemichordate mitochondria. Proc Natl Acad Sci USA 95:37033707.

Castresana J, Feldmaier-Fuchs G, Yokobori S, Satoh N, Pääbo S, 1998b. The mitochondrial genome of the hemichordata Balanoglossus carnosus and the evolution of deuterostome mitochondria. Genetics 150:1115-1123.

Ehlers U, 1991. Comparative morphology of statocysts in the platyhelminthes and the xenoturbellida. Hydrobiologia 227:263-271. 
Ehlers U, Sopott-Ehlers B, 1997a. Xenoturbella bocki: organisation and phylogenetic position as sister taxon of the Bilateria. Verh Dtsch Zool Ges 90:168-.

Ehlers U, Sopott-Ehlers B, 1997b. Ultrastructure of the subepidermal musculature of Xenoturbella bocki, the adelphotaxon of the Bilateria. Zoomorphology 117:7179.

Fitch DH, 2005. Introduction to nematode evolution and ecology. In: The C. elegans Research Community, editor, WormBook. Doi/10.1895/wormbook.1.19.1 (August 31, 2005).

Franzén Å, Afzelius BA, 1987. The ciliated epidermis of Xenoturbella bocki (Platyhelminthes, Xenoturbellida) with some phylogenetic considerations. Zool Scripta 16:9-17.

Fritzsch G, Schlegel M, Stadler P, 2006. Alignments of mitochondrial genome arrangements: Applications to metazoan phylogeny. J Theor Biol 240:511-520.

Himeno H, Masaki H, Kawai T, Ohta T, Kumagai I, Miura K, Watanabe K, 1987. Unusual genetic codes and a novel gene structure for tRNA(AGYSer) in starfish mitochondrial DNA. Gene 56:219-230.

Hofacker IL, Fontana W, Stadler PF, Bonhoeffer LS, Tacker M, Schuster P, 1994. Fast folding and comparison of RNA secondary structures. Monatsh Chem 125:167-188.

Israelsson O, 1997. ... and molluscan embryogenesis. Nature 390:32.

Israelsson O, 1999. New light on the enigmatic Xenoturbella (phylum uncertain): ontogeny and phylogeny. Proc Royal Soc London Ser B 266:835-841.

Israelsson O, Budd GE, 2005. Eggs and embryos in Xenoturbella (phylum uncertain) are not ingested prey. Dev Genes Evol 215:358-363.

Jägersten $G, 1959$. Further remarks on the early phylogeny of metazoa. Zoologiska Bidrag 33:79-108.

Lartillot N, Philippe H, 2004. A Bayesian mixture model for across-site heterogeneities in the amino-acid replacement process. Mol Biol Evol 21:1095-1109.

Lartillot N, Philippe H, 2006. Computing Bayes factors using thermodynamic integration. Syst Biol 55:195-207.

Lavrov DV, Brown WM, 2001. Trichinella spiralis mtDNA: A nematode mitochondrial genome that encodes a putative ATP8 and normally structured tRNAs and has a gene arrangement relatable to those of coelomate metazoans. Genetics 157:621-637.

Lavrov DV, Lang BF, 2005. Poriferan mtDNA and animal phylogeny based on mitochondrial gene arrangements. Systematic Biol 54:651-659.

Lowe TM, Eddy SR, 1997. tRNAscan-SE: a program for improved detection of transfer RNA genes in genomic sequence. Nucleic Acids Res 25:955-964.

Lundin K, 1998. The epidermal ciliary rootlets of Xenoturbella bocki (Xenoturbellida) revisited: new support for a possible kinship with the acoelomorpha (platyhelminthes). Zoologica Scripta 27:263-270.

Nohara M, Nishida M, Miya M, Nishikawa T, 2005. Evolution of the mitochondrial genome in cephalochordata as inferred from complete nucleotide sequences from two Epigonichthys species. J Mol Biol 60:526-537.

Noren M, Jondelius U, 1997. Xenoturbella's molluscan relatives. Nature 390:31-32.

Notredame C, Higgins D, Heringa J, 2000. T-Coffee: A novel method for multiple sequence alignments. J Mol Biol 302:205-217.

Pedersen KJ, Pedersen LR, 1986. Fine structural observations on the extracellular matrix (ECM) of Xenoturbella bocki Westblad, 1949. Acta Zool 67:103-113.

Pedersen KJ, Pedersen LR, 1988. Ultrastructural observations on the epidermis of Xenoturbella bocki Westblad, 1949; with a discussion of epidermal cytoplasmic filament systems of invertebrates. Acta Zool 69:231-246.

Raikova OI, Reuter M, Jondelius U, Gustafsson MKS, 2000. An immunocytochemical and ultrastructural study of the nervous and muscular systems of Xenoturbella westbladi (bilateria inc. sed.). Zoomorphology 120:107-118.

Reisinger E, 1960. Was ist Xenoturbella? Zeitschrift f Wissenschaftliche Zoologie 164:188-198.

Rieger RM, Tyler S, Smith III JPS, Rieger G, 1991. Platyhelminthes: Turbellaria. In: Harrison FW, Bogitsh BJ, editors, Microscopic anatomy of invertebrates. Volume 3: Platyhelminthes and Nemertinea, (pp. 7-140). New York: Wiley-Liss.

Rohde K, Watson N, Cannon LRG, 1988. Ultrastructure of epidermal cilia of Pseudactinoposthia sp. (Platyhelminthes, Acoela): implications for the phylogenetic status of the Xenoturbellida and Acoelomorpha. J Submicrosc Cytol Pathol 20:759-767.

Ronquist F, Huelsenbeck JP, 2003. MrBayes 3: Bayesian phylogenetic inference under mixed models. Bioinformatics 19:1572-1574.

Ruiz-Trillo I, Paps J, Loukota M, Ribera C, Jondelius U, Baguñà J, Riutort M, 2002. A phylogenetic analysis of myosin heavy chain type II sequences corroborates that Acoela and Nemertodermatida are basal bilaterians. Proc Natl Acad Sci USA 99:11246-11251.

Ruiz-Trillo I, Riutort M, Fourcade HM, Bagunñà J, Boore JL, 2004. Mitochondrial genome data support the basal position of Acoelomorpha and the polyphyly of the Platyhelminthes. Mol Phylog Evol 33:321-332.

Sankoff D, Leduc G, Antoine N, Paquin B, Lang BF, 1992. Gene order comparisons for phylogenetic inference: Evolution of the mitochondrial genome. Proc Natl Acad Sci USA 89:6575-6579.

Schmidt HA, Strimmer K, Vingron M, von Haeseler A, 2002. TREEPUZZLE: maximum likelihood phylogenetic analysis using quartets and parallel computing. Bioinfor- 
matics 18:502-504.

Stach T, Dupont S, Isrealson O, Fauville G, Nakano H, Kånneby T, horndyke M, 2005. Nerve cells of Xenoturbella bocki (phylum uncertain) and Harrimania kupfferi (enteropneusta) are positively immunoreactive to antibodies raised against echinoderm neuropeptides. J Marine Biol Association UK 85:1519-1524.

Swofford DL, 2002. PAUP*: Phylogenetic Analysis Using Parsimony (*and Other Methods) Version 4.0b10. Sunderland, MA: Sinauer Associates. Handbook and Software.

Telford MJ, Lockyer AE, Cartwright-Finch C, Littlewood DT, 2003. Combined large and small subunit ribosomal RNA phylogenies support a basal position of the acoelomorph flatworms. Proc Biol Sci 270:1077-1083.

Westblad E, 1949. Xenoturbella bocki n.g., n.sp., a peculiar, primitive Turbellarian type. Arkiv for Zoologi 1:3-29.

Yokobori S, Oshima T, Wada H, 2005. Complete nucleotide sequence of the mitochondrial genome of Doliolum nationalis with implications for evolution of urochordates. Mol Phylogenet Evol 34:273-283.

Yokobori SI, Watanabe Y, Oshima T, 2003. Mitochondrial genome of Ciona savignyi (urochordata, ascidiacea, enterogona): comparison of gene arrangement and tRNA genes with Halocynthia roretzi mitochondrial genome. J Mol Evol 57:574-587.

Zrzavy J, Mihulka S, Kepka P, Bezdek A, Tietz D, 1998. Phylogeny of the metazoa based on morphological and 18S ribosomal DNA evidence. Cladistics 14:249-285. 